Trauma Berufskrankh 2008 10 [Suppl 2]:167-174

DOI 10.1007/s10039-007-1306-4

Online publiziert: 7. März 2008

๑) Springer Medizin Verlag 2008

J. Hardes · G. Gosheger

Klinik und Poliklinik für Allgemeine Orthopädie, Universitätsklinikum Münster, Münster

\title{
Die pathologische Fraktur aus Sicht des Orthopäden und Unfallchirurgen
}

tumor nicht nachgegangen, kann sich im weiteren Verlauf aufgrund des Knochensubstanzverlusts und der strukturellen Schwächung des Knochens eine pathologische Fraktur entwickeln. Diese ist als eine Fraktur ohne adäquates Trauma definiert; ein klinisches Indiz ist daher häufig die nur geringgradige oder fehlende Mitverletzung der umgebenden Weichteile [12]. Nicht selten ereignet sich jedoch eine pathologische Fraktur ohne vorherige Malignomanamnese. Insbesondere bei diesen Patienten müssen diagnostische und therapeutische Automatismen greifen, um erstens die stattgehabte Fraktur auch als pathologische zu erkennen und zweitens nicht in einen verfrühten chirurgischen Aktionismus zu verfallen.

\section{Diagnostischer Algorithmus} dingt durch den medizinischen Fortschritt - die Patienten selbst mit Metastasen noch eine längere Überlebenszeit aufweisen $[3,11,12]$. Schon jetzt können $\mathrm{Pa}$ tienten mit Nierenkarzinommetastasen (insbesondere bei Vorliegen von Spätmetastasen) durchaus über 5 Jahre nach der Erstdiagnose noch am Leben sein [1].

Prädilektionsstellen für das Auftreten von Knochenmetastasen sind [3]

- die Wirbelsäule,

- die Beckenknochen,

- Femur,

- Rippen und

- Humerus.

Wird dem Leitsymptom einer Knochenmetastase in Form des Instabilitätsschmerzes (insbesondere Wirbelsäule, Becken und untere Extremität) von Patient und Arzt bei schon bekanntem Primär-

\section{Bekannter Primärtumor}

Relativ leicht zu diagnostizieren sind pathologische Frakturen bei einem bekannten Primärtumor. Dennoch können sich auch hierbei Schwierigkeiten ergeben. Zum einen muss die Anamnese onkologische Vorerkrankungen gründlich abfragen, da einige Patienten dazu tendieren, diese zu verschweigen. Zum anderen ist bei einer onkologischen Vorerkrankung diese nicht immer für eine strukturelle Schwächung verantwortlich. So kann es durchaus Komorbiditäten wie z. B. die Osteoporose geben, welche für die Fraktur verantwortlich sind. Des Weiteren sind postradiogene Knochensarkome Jahre nach der Primärtumorerkrankung keine Seltenheit und bedürfen einer weiten Tumorresektion gemäß Enneking [6] (z. B. Osteosarkom des proximalen $\mathrm{Hu}$ merus nach Bestrahlung der Axilla bei einem Mammakarzinom). Auch kann es vorkommen, dass bei einem Patienten mit einer Karzinomanamnese die neu aufgetretene pathologische Fraktur durch einen anderen malignen (z. B. Lymphom, primäres Knochensarkom) oder gar benignen, aggressiven Knochentumor bedingt ist [11]. Würde zum Beispiel bei einer Patientin mit einem Mammakarzinom und einer erstmalig eingetretenen pathologischen Fraktur des proximalen Femur nach einer intramedullären Stabilisierung ohne vorherige histologische Abklärung retrospektiv ein Chondrosarkom diagnostiziert, so hätte die Patientin eigentlich einer weiten Tumorresektion des proximalen Femurs zugeführt werden müssen. Durch die jetzt erfolgte Kontamina- 


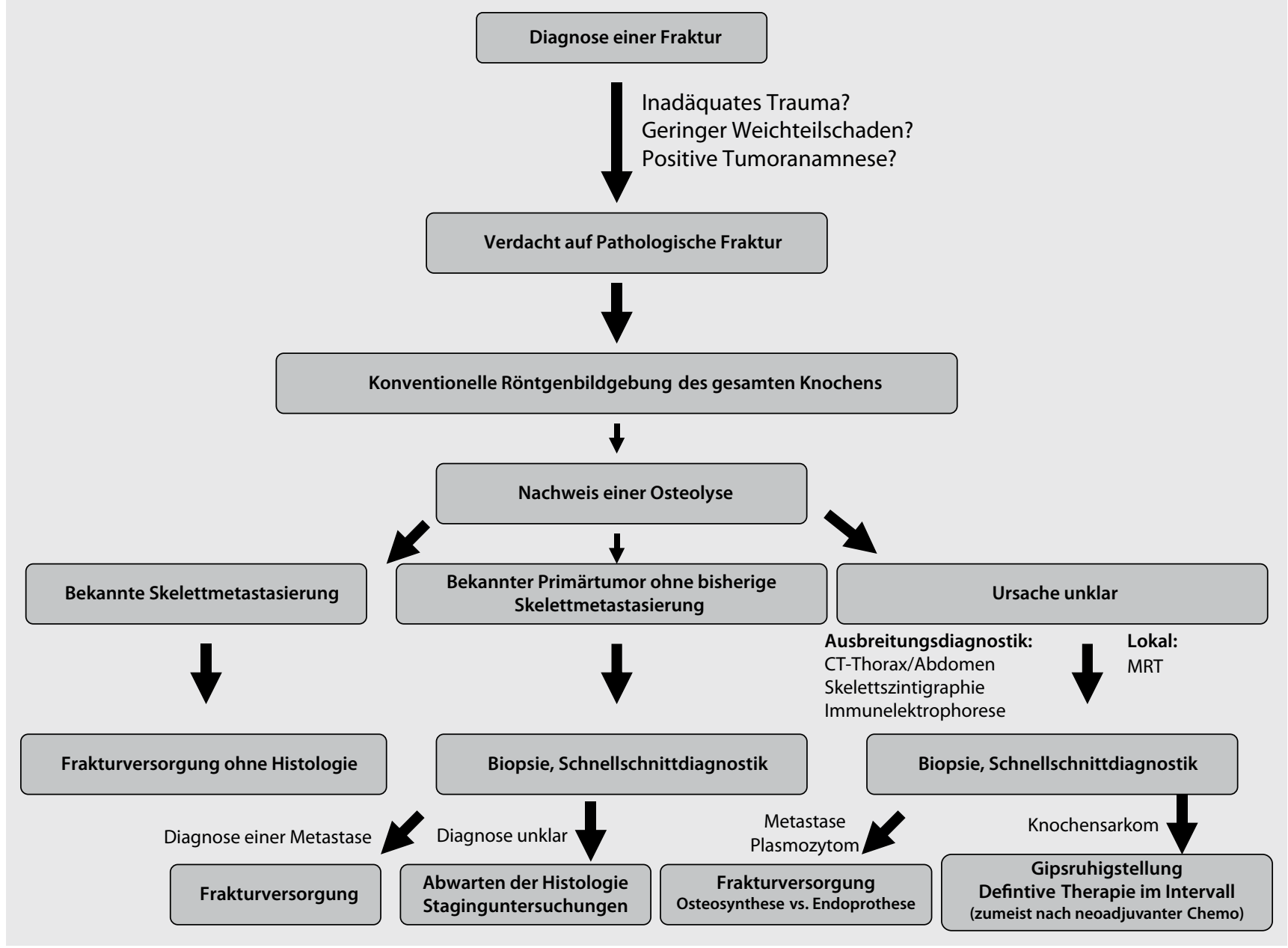

Abb. $1 \Delta$ Diagnostischer Algorithmus bei klinischem Verdacht auf eine pathologische Fraktur

tion des gesamten Femurs muss nun ein totaler Femurersatz durchgeführt werden. Bei Vorliegen eines glutealen Hämatoms oder eines solchen im Bereich der Verriegelungsschrauben muss gegebenenfalls über eine Hüftexartikulation diskutiert werden. Daher sollte selbst bei einem bekannten Primärtumor ohne dokumentierte Skelettmetastasierung vor der eigentlichen operativen Versorgung noch eine intraoperative Schnellschnittuntersuchung durchgeführt werden [11]. Liegt jedoch eine bekannte Skelettmetastasierung vor, kann aus unserer Sicht auf eine nochmalige histologische Sicherung verzichtet werden, wenn röntgenographisch eine Skelettmetastase nachgewiesen ist ([11], • Abb. 1).

\section{Erstmanifestation einer Tumorerkrankung}

Nicht selten erfolgt eine pathologische Fraktur als Erstmanifestation einer Tumo- rerkrankung. Bei Verdacht auf eine pathologische Fraktur bei Patienten ohne eine Malignomanamnese sollte vor der operativen Intervention zunächst eine lokale und auch Ausbreitungsdiagnostik veranlasst werden ( $\bullet$ Abb. 1). Hierzu zählt neben einem konventionellen Röntgenbild in zwei Ebenen auch die Magnetresonanztomographie des betroffenen Skelettabschnitts, um die intra- und extraossäre Tumorausdehnung sicher zu erfassen. Hier sollte ein Augenmerk insbesondere darauf gelegt werden, ob die Weichteilkomponente an Gefäß-/Nervenstraßen heranreicht. Diese Information ist bei einer eventuell später notwendig werdenden weiten Resektion gemäß Enneking [6] von großer Bedeutung. Die Röntgenbildgebung sollte unbedingt den gesamten betroffenen Knochen erfassen, um eventuelle Zweitläsionen zu erkennen [11]. Es wäre z. B. fatal, eine Verbundosteosynthese des proximalen Femurs direkt oberhalb einer zweiten, noch nicht frak- turierten Metastase des distalen Femurs enden zu lassen. Bei Frakturen des proximalen Femurs, bei denen ein endoprothetischer Ersatz angestrebt wird, muss eine Metastase des Acetabulums ausgeschlossen werden. Sollte hier konventionell-radiographisch eine Metastase nachgewiesen werden, empfiehlt es sich, mittels Schnittbilddiagnostik die Ausdehnung der Läsion zu bestimmen $[3,11]$.

\section{Ausbreitungsdiagnostik}

Die 3-Phasen-Skelettszintigraphie dient zum einen der Aktivitätseinschätzung des betroffenen Skelettabschnitts (dabei ist die Befundverzerrung durch die Fraktur zu beachten), zum anderen ermöglicht sie den Nachweis weiterer ossärer Läsionen im Rahmen der Ausbreitungsdiagnostik. Eine Computertomographie des Thorax und Abdomens kann entweder dem Primärtumor- oder Metastasennachweis dienen. Bei Verdacht auf eine 
Mammakarzinomerkrankung kann eine Mammographie eine weitere diagnostische Hilfestellung geben. Besteht der Verdacht auf eine hämatopoetische Erkrankung (häufige Ursache ist das Plasmozytom), kann schon präoperativ ein Differentialblutbild, eine Eiweißelektrophorese und eine Urinuntersuchung auf BenceJones-Eiweißkörper erfolgen [11]. Ergibt sich hierbei ein Hinweis auf ein Plasmozytom, sollte ein konventionell-radiographischer Plasmozytomstatus (Röntgenographie von Humerus, Femur, Schädel, Lenden- und Brustwirbelsäule) erfolgen, da die Skelettszintigraphie häufig fälschlicherweise negative Befunde (etwa 20\%) ergibt [11]. Bei Verdacht auf eine Prostatakarzinommetastase ist zudem ein PSATest hilfreich. Diese umfangreiche Diagnostik muss innerhalb weniger Tage zu absolvieren sein und die Fraktur des Patienten muss in dieser Zeit ruhig gestellt werden. Je nach Frakturlokalisation geschieht dies in einer Armschlinge, einem Gips oder auch im Rahmen einer Bettruhe. Um eine (schmerzhafte) Einstauchung der Frakturenden zu vermeiden, verwenden wir bei Frakturen der unteren Extremität eine Weichteilextension. Die Schmerzfreiheit des Patienten muss des Weiteren durch eine adäquate Analgesie gewährleistet werden.

Die zuvor beschriebene Ausbreitungsdiagnostik liefert bei der Mehrzahl der Patienten schon eine klare Verdachtsdiagnose, Informationen über das Krankheitsstadium und damit auch eine gewisse $\mathrm{Ab}$ schätzung der Prognose. Damit kann der Chirurg in der darauf folgenden Operation das Stabilisierungsverfahren auswählen, das in der individuellen Situation am geeignetsten erscheint. Auch muss bedacht werden, dass einige Tumorentitäten (z. B. Nierenkarzinom, Plasmozytom) mit einer verstärkten Blutungsneigung vergesellschaftet sind. Besteht zum Beispiel nach der Computertomographie des Abdomens der Verdacht auf eine Nierenkarzinommetastase, so ist eine starke Blutung bei einem intraläsionalen Vorgehen sehr wahrscheinlich. Diese Tumorblutungen sind im nekrotischen Tumorgewebe chirurgisch häufig nur schwer zu stillen und sollten daher vermieden werden. In diesen Fällen ist deshalb eine prä-

Trauma Berufskrankh 2008 · 10[Suppl 2]:167-174 DOI 10.1007/s10039-007-1306-4

(c) Springer Medizin Verlag 2008

\section{J. Hardes. G. Gosheger \\ Die pathologische Fraktur aus Sicht des Orthopäden und Unfallchirurgen}

\section{Zusammenfassung}

Aufgrund der demografischen Entwicklung nimmt die Zahl der Krebserkrankungen in Deutschland stetig zu. Während primäre Knochentumoren selten sind, steigt die Zahl der Skelettmetastasen deutlich an. Bei zu spät entdeckten Knochenmetastasen sind pathologische Frakturen relativ häufig und betreffen vornehmlich die Wirbelsäule, das Becken, Femurknochen und den Humerus. Der vorliegende Artikel gibt einen Überblick über die adäquaten diagnostischen Schritte bei Verdacht auf Vorliegen einer pathologischen Fraktur sowie die unterschiedlichen Therapiestrategien. Art und Ausmaß der Therapie richten sich unter anderem nach der Entität, dem Zeitpunkt des Auftretens der Metasta- sen, dem Erkrankungsstadium und der Lokalisation der Fraktur. Ziel der operativen Therapie sollte es sein, eine bis zum Tod des $\mathrm{Pa}$ tienten belastungsstabile Extremität wiederherzustellen. Revisionsoperationen aufgrund von Tumorprogress, Pseudarthrosen und/oder Materialversagen sind auf ein Minimum zu beschränken, da sie die Lebensqualität in der palliativen Gesamtsituation beeinträchtigen.

\section{Schlüsselwörter}

Pathologische Fraktur - Knochenmetastase . Osteosynthese - Endoprothese .

Tumorerkrankung

\section{The pathologic fracture from the viewpoint of the orthopaedic specialist and trauma surgeon}

\section{Abstract}

Owing to the demographic development, the number of patients in Germany with cancer has been steadily rising over recent decades. While primary bone tumours remain rare, the number of skeletal metastases is clearly increasing. When diagnosis of skeletal metastasis is late, pathologic fractures are not uncommon; prediliction sites for fracture are the vertebral column, the pelvis, the femur and the humerus. This review gives an overview of the necessary diagnostic steps when a pathologic fracture is suspected and of the different treatment strategies. The type and extent of the treatment depends for exam- ple on the tumour entity, the time of occurrence of skeletal metastasis, the stage of disease and the site of the fracture. The aim of surgical treatment is full weight-bearing on the involved extremity until the patient's death. Revision surgery due to tumour progression, pseudarthrosis and / or implant failures should be avoided because of an additional limitation of life quality in the palliative situation.

\section{Keywords}

Pathologic fracture - Bone metastasis · Osteosynthesis · Endoprosthesis - Tumour disease 
operative Embolisation der großen tumorzuführenden Gefäße zu empfehlen [3].

\section{Durchführung der Gewebebiopsie}

Die Biopsie des Knochens muss immer unter der Annahme erfolgen, dass die histologische Aufarbeitung einen primären Knochentumor ergibt. Bei dieser Diagnose muss aufgrund einer möglichen Tumorzellkontamination bei der späteren weiten Tumorresektion der Biopsietrakt mit entfernt werden. Deshalb sollte ein Biopsiezugang durch Gelenke, an Gefäß/Nervenstraßen und durch Muskelkompartimente, welche nur schlecht verzichtbar sind, vermieden werden. Bei einer Tumorzellkontamination eines Gelenks muss dieses en bloc entfernt werden, eine ausgedehnte Kontamination von Gefäß-/ Nervenstraßen hat eine Amputation zur Folge. Eine Biopsie durch den Musculus rectus femoris würde eine aufgehobene Streckung des Kniegelenks bedeuten. So liegt zum Beispiel der klassische Biopsiezugang am Femur anterolateral über dem Musculus vastus lateralis. Weitherhin muss bei der Gewebeentnahme beachten werden, dass möglicht viel Gewebe gewonnen wird. Dieses ist insbesondere bei bereits nekrotisch zerfallenen Tumoren wichtig, da der Pathologe ausreichend repräsentatives Gewebe benötigt.

\section{Einschätzung der Prognose}

Die Einschätzung der Prognose eines Patienten mit einer Knochenmetastasierung ist schwierig und individuell selbstverständlich höchst unterschiedlich [13]. In einer Studie von Nathan et al. [13] wurde versucht, die Überlebenszeit nach der Diagnose von einer oder mehreren Knochenmetastasen einzuschätzen. Gelungen ist dies trotz einer Vielzahl von berücksichtigten Faktoren nur bei 33 von $181 \mathrm{~Pa}$ tienten (18\%) mit einer 20\%igen Standardabweichung. Bei 43\% der Patienten wurde retrospektiv gesehen eine zu kurze Lebenserwartung zugesprochen, bei $39 \%$ eine zu lange. Dennoch muss bei Vorliegen einer pathologischen Fraktur anhand einer Vielzahl von Faktoren versucht werden, eine ungefähre Einschätzung der Prognose vorzunehmen, um das geeignete Operationsverfahren zu wählen. Da- bei gilt zum einen, dass das gewählte Osteosyntheseverfahren beziehungsweise der endoprothetische Ersatz den Patienten „überleben“ muss, um belastende Revisionsoperationen zu vermeiden [3]. Zum anderen sollte bei Patienten mit einer stark reduzierten Prognose eine belastende „Übertherapie“ (z. B. in Form einer Endoprothese) ebenfalls unterbleiben.

Die Scandinavian Sarcoma Group Skeletal Metastasis [8] untersuchte multizentrisch prospektiv 460 Patienten (Durchschnittsalter 64 Jahre) mit einer oder mehreren Knochenmetastasen bezüglich der Überlebenszeit nach operativer Therapie der Metastasen (mit oder ohne Fraktur). Hierbei ergab sich, dass die Überlebensrate postoperativ nach 1 Jahr $40 \%$, nach 2 Jahren 30\% und nach 3 Jahren $20 \%$ betrug. Eine multivariate Regressionsanalyse zeigte, dass das Vorliegen einer pathologischen Fraktur, einer simultanen viszeralen Metastasierung, eines Hämoglobinwerts von $<7 \mathrm{mmol} / \mathrm{l}$ bzw. eines Bronchialkarzinoms negative Prädiktoren für das Überleben darstellten. Auch in der Studie von Nathan et al. [13] war die Diagnose eines Bronchialkarzinoms ein statistisch signifikanter negativer Prädiktor (mittlere Überlebenszeit von 4 Monaten nach der Operation der Knochenmetastase).

Wedin et al. [14] ermittelten, dass Patientinnen mit einem Mammakarzinom bei Auftreten von Knochenmetastasen noch eine mittlere Überlebenszeit von 22 Monaten aufwiesen, verglichen mit nur 15 Monaten bei Patientinnen, die neben den Metastasen auch ein Lokalrezidiv aufzuweisen hatten. Die schlechteste Prognose (12 Monate) hatten Frauen mit multiplen Metastasen oder Rezidiven. Das simultane Vorliegen von viszeralen Metastasen bei Patientinnen mit einem Mammakarzinom ist mit einer weiteren Prognoseverschlechterung behaftet [4]. Coleman et al. [4] kamen zu dem Ergebnis, dass Patientinnen mit simultanen Leber- und Knochenmetastasen nur noch eine mediane Überlebenszeit von 5,5 Monaten hatten. Dürr et al. [5] ermittelten für Patienten mit einem Nierenkarzinom und Knochenmetastasen eine Gesamtüberlebenswahrscheinlichkeit von $49 \%$ nach $1 \mathrm{Jahr}$, $39 \%$ nach 2 Jahren, $25 \%$ nach 3 Jahren und $15 \%$ nach 5 Jahren. Bei getrennter Betrachtung von Patienten mit einer singulären
Knochenmetastasierung ergab sich eine 5Jahres-Überlebenswahrscheinlichkeit von 28\%. Nur das Krankheitsstadium und der Zeitpunkt der Metastasierung hatten einen signifikanten Einfluss auf die Überlebenszeit. Die beste Prognose mit einer 5Jahres-Überlebenswahrscheinlichkeit von $54 \%$ hatten Patienten mit einer singulären Metastase ab dem 2. Jahr nach der Erstdiagnose. Patienten, die keines dieser Kriterien erfüllten, zeigten nach 5 Jahren eine Überlebenswahrscheinlichkeit von o\%.

Auch Nathan et al. [13] konnten anhand von 191 Patienten mit Knochenmetastasen statistisch signifikant zeigen, dass bei singulärer Knochenmetastase das mittlere Überleben 24,7 Monate und bei Vorliegen von mehreren Knochenmetastasen nur 6 Monate betrug. Im Fall einer viszeralen Metastasierung (Lunge und Abdomen) war die mittlere Überlebenszeit ebenfalls signifikant geringer (6 Monate) gegenüber Patienten ohne viszerale Metastasen (durchschnittliche Überlebenszeit von 24,7 Monaten). Auch in dieser Studie war ein niedriger Hämoglobinwert im Sinne einer Tumoranämie ein signifikanter negativer Prädiktor.

\section{Wahl des Operationsverfahrens}

Bei der Wahl des geeigneten Operationsverfahrens muss also zunächst die Entität, das anhand der Ausbreitungsdiagnostik ermittelte Krankheitsstadium und damit einhergehend die Prognose des Patienten berücksichtigt werden.

Ergibt sich in der histologischen Schnellschnittdiagnostik ein maligner primärer Knochentumor (z. B. Chondrosarkom, Osteosarkom, Ewing-Sarkom), so muss die Operation beendet und die betreffende Extremität im Gips ruhig gestellt werden [12]. Bei chemosensiblen Tumoren wie dem Osteo- und Ewing-Sarkom erfolgt dann zunächst eine neoadjuvante Chemotherapie und daran anschließend die weit ins Gesunde reichende Tumorresektion [12]. Eine Frakturversorgung bzw. ein „Anoperieren“ würde zu einer Tumorzellverschleppung führen und die extremitätenerhaltende Therapie nach Abschluss der Chemotherapie erschweren oder gar unmöglich machen [12]. In der Regel ist bei Vorliegen einer pathologischen Fraktur eine extremitätenerhaltende Therapie 
nicht mit einer erhöhten Rate an Lokalrezidiven assoziiert [2]. Kann sich der Pathologe anhand der Schnellschnittuntersuchung nicht sicher auf eine Entität und Dignität festlegen, muss die endgültige Histologie ebenfalls zunächst abgewartet werden.

Bei der Diagnose einer Knochenmetastase muss das Operationsverfahren unter anderem von der Prognose des Patienten abhängig gemacht werden. Eine weite Tumorresektion und Implantation einer Tumorprothese ist bei Patienten $\mathrm{zu}$ bevorzugen, die eine gute Prognose aufweisen (insbesondere singuläre Spätmetastasen bei Mamma- und Nierenkarzinom) $[4,5,11]$. Neben der Prognoseverbesserung, die die Patienten hierdurch erfahren, ist mit diesem Vorgehen auch am ehesten sichergestellt, dass es auch nach Jahren zu keinem lokalen Tumorprogress kommen wird [3]. Ob nach einer weiten Metastasenresektion gemäß Enneking [6] eine adjuvante Strahlentherapie erforderlich ist, kann durch Literaturdaten nicht belegt werden. Wir selbst führen sie nach einer weitaus häufigeren marginalen Resektion nach Enneking [6] in der Regel durch und verzichten nur bei einer weit im Gesunden erfolgten Resektion auf die adjuvante Strahlentherapie.

Liegt eine deutlich eingeschränkte Lebenserwartung vor, sollte eine intraläsionale Resektion erfolgen und nach Möglichkeit auf die Implantation einer Tumorendoprothese verzichtet werden. Ausnahmen können bei ausgedehnten Nierenkarzinommetastasen bestehen, welche nach der Embolisation eine noch ausgeprägte Vaskularisation zeigen. Die Indikation einer weiten, extrafokalen Resektion ist hier zwar nicht onkologisch gegeben, kann jedoch die intraoperative Blutung bei intraläsionaler Resektion drastisch reduzieren. Des Weiteren kann trotz intraläsionalem Vorgehen bei einem ausgedehnten Knochensubstanzverlust, bei dem auch eine konventionelle Endoprothese nicht mehr ausreicht, die Implantation einer Tumorprothese notwendig werden.

Faktoren, von denen die Wahl des geeigneten Osteosyntheseverfahrens abhängt, sind unter anderem:

- die Prognose der Patienten

- die Lokalisation der Fraktur,

- die Gelenknähe der Fraktur,
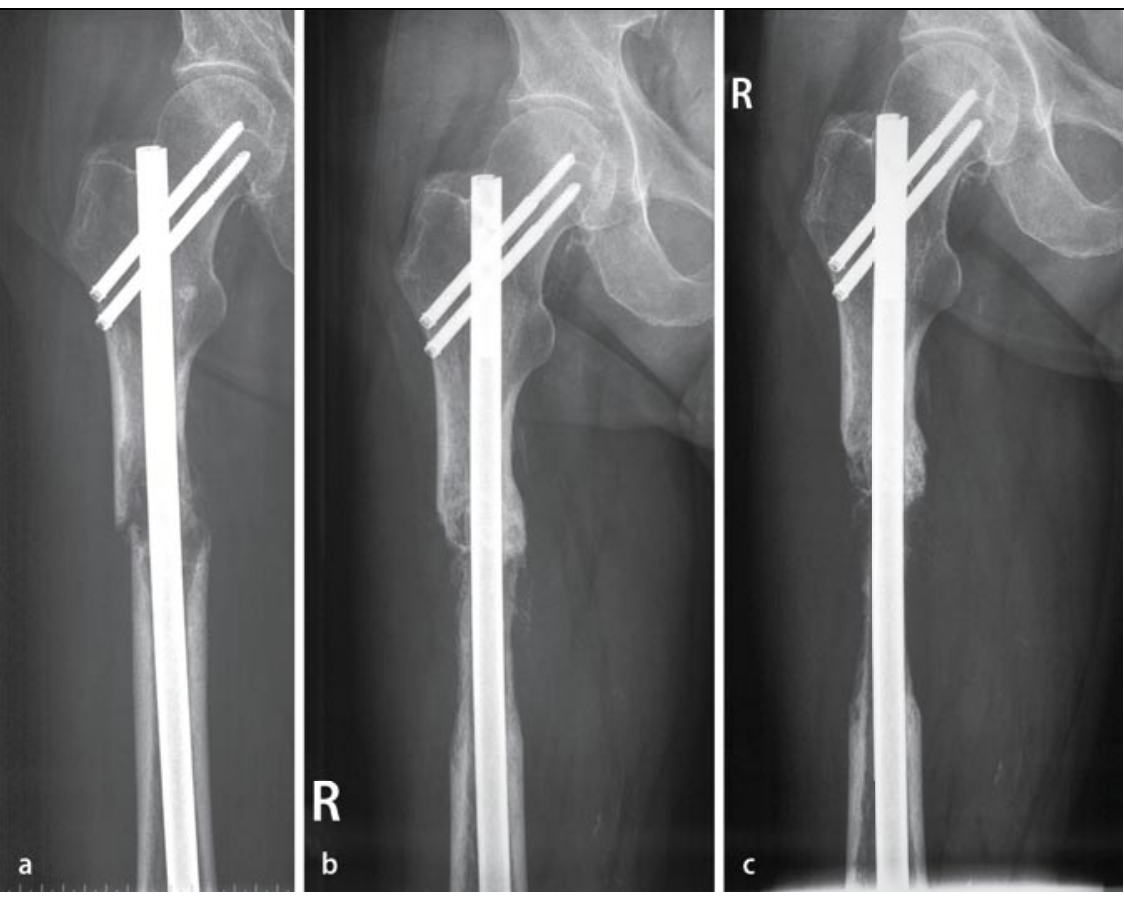

Abb. $2 \Delta$ a Postoperatives anterior-posteriores Röntgenbild nach pathologischer Fraktur des rechten Femurs bei einem 64-jährigen Patienten mit einem multipel metastasierten Nierenkarzinom, b 4 Jahre postoperativ beginnende Knochenresorption um den Marknagel herum: Lokalrezidiv trotz adjuvanter Strahlentherapie mit 45 Gray, c Progress der Knochenresorption 4,5 Jahre postoperativ trotz zwischenzeitlich erfolgter erneuter Strahlentherapie mit Aufsättigung der Dosis bis 65 Gray
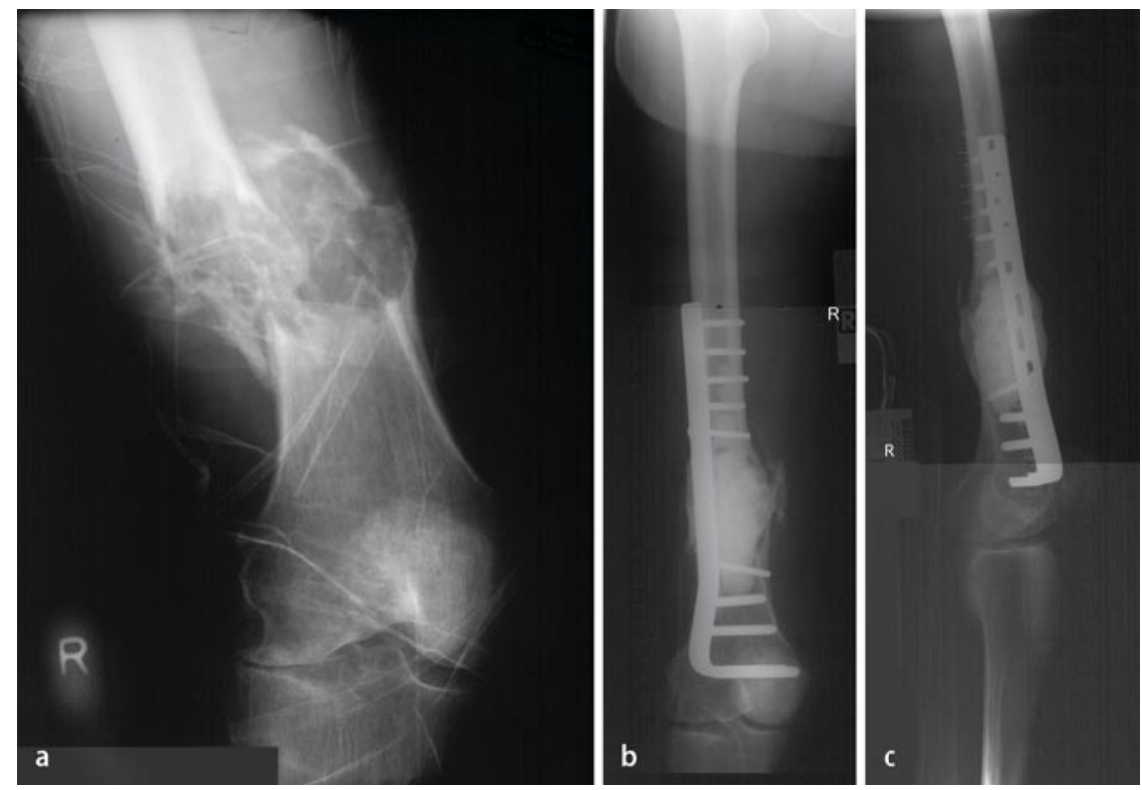

Abb. $3 \Delta$ a 75-jähriger Patient mit einer pathologischen Fraktur bei ausgedehnter Osteolyse eines Bronchialkarzinoms, b, c postoperative Röntgenbilder der Verbundosteosynthese mit einer Klingenplatte

- der Knochensubstanzverlust und

- die Strahlensensibilität des Tumors.

Bei Vorliegen eines relativ strahlenresistenten Tumors und einer längeren Restüberlebenswahrscheinlichkeit des $\mathrm{Pa}$ tienten muss die intramedulläre Tumor- zellkontamination bedacht werden. Ein Lokalrezidiv ist hierbei wahrscheinlicher und kann dann den gesamten Knochen involvieren (- Abb. 2a-c).

Plattenosteosynthese. Diese kann bei metaepiphysären Osteolysen sehr effektiv 

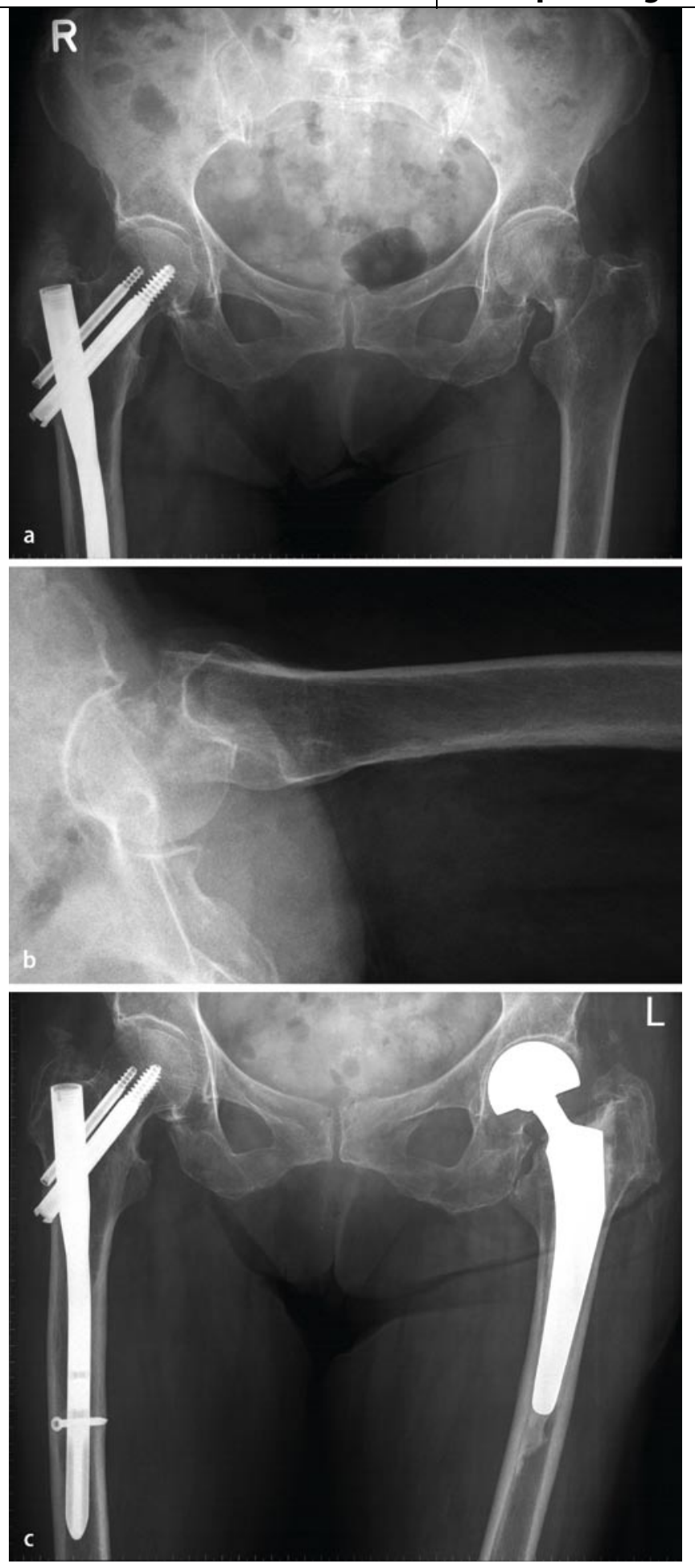

eingesetzt werden, vorausgesetzt, das Gelenk ist nicht in Mitleidenschaft gezogen und es besteht ausreichend Knochensubstanz zur Fixation der Platte. Wir verwenden eine Verbundosteosynthese mit einer Platte häufiger bei Frakturen der distalen Femurmetaphyse als bei proximalen Femurfrakturen ( $\bullet$ Abb.3a-c). Bei Patienten mit einer zu erwartenden län-
Abb. $4<$ a, b Röntgenbild des Beckens und Lauenstein-Projektion links einer 85jährigen Patientin mit einem multiplen Myelom: Zustand nach Marknagelimplantation rechts bei proximaler Femurfraktur, c postoperatives Röntgenbild nach Implantation einer zementierten Hemialloarthroplastik

Marknagelosteosynthese $\mathrm{zu}$ bevorzugen (dabei muss die Strahlensensibilität des Primärtumors bedacht werden). Je mehr Knochensubstanz zum benachbarten Gelenk zur Verfügung steht desto rigider ist die Stabilisierung. Es sollten sämtliche Verriegelungsoptionen ausgeschöpft werden, da eine dauerhafte Pseudarthrose möglich ist und das Implantat bis zum Tod des Patienten die gesamte Last der betreffenden Extremität übernehmen muss (- Abb. 2a-c, [3]). Im Bereich des proximalen Femurs sollten die Verriegelungen unbedingt den Schenkelhals und Hüftkopf miterfassen [11]. Des Weiteren sollte der Marknagel den gesamten Knochen stabilisieren, da es nicht selten ist, dass im Krankheitsverlauf weitere Osteolysen des Knochens auftreten [11]. Eine retrograde Femurmarknagelung bei distalen Femurfrakturen sollte unterbleiben, da hierbei zum einen die Nagelinsertion eine Kontamination des Kniegelenks nach sich zieht und zum anderen nicht das gesamte Femur prophylaktisch stabilisiert werden kann [11].

Bei der Wahl des Operationsverfahrens ist zu berücksichtigen, dass der tumorkontaminierte Knochen häufig keine Frakturheilung zeigt $[3,14]$. Dies hat seinen Grund nicht nur in der Tumorerkrankung an sich, sondern auch in der meistens durchgeführten (neo-)adjuvanten Chemo- und insbesondere Strahlentherapie $[11,14]$. So ermittelten Gainor u. Buchert [7], dass nach einer pathologischen Fraktur in 50\% der Fälle keine Frakturheilung mehr eintritt. Bei Patientinnen mit einem Mammakarzinom erfolgte eine Frakturheilung sogar nur in $37 \%$ der Fälle. Daher ist bei der Wahl der Operationsmethode zu bedenken, dass die Osteosynthese die komplette Lastübertragung auf die betroffene Extremität quasi für den Rest des Lebens ermöglichen soll. Eine Reduzierung der Belastungsfähigkeit aufgrund fehlender Frakturheilung würde die Lebensqualität in dieser palliativen Therapiesituation zusätzlich einschränken [11, 14]. gidere, winkelstabile Implantate. Ob sich hierdurch die Rate an Materialversagen reduzieren lässt, kann noch nicht bewertet werden.

Marknagelosteosynthese. Aus unserer Sicht ist bei Vorliegen einer pathologischen Fraktur der Metadiaphyse die
Knochenzement. Ein bedeutender Fortschritt in der Behandlung pathologischer Frakturen konnte durch die Einführung des Knochenzements gemacht werden, da mit diesem auch ohne Frakturheilung ausgedehnte Defekte rekonstruiert wer- 
den können [11]. Ferner kann nun eine Kürettage des Tumors im Sinne eines Tumordebulkings erfolgen, sodass der Resttumor besser auf die adjuvante Strahlentherapie anspricht und die Tumorzellverschleppung bei einer intramedullären Stabilisierung geringer ausfällt. Die exotherme Polymerisation des Knochenzements übt einen zusätzlichen tumortoxischen Effekt aus [3]. Eine Tumorkürettage ist somit sowohl vor einer Plattenosteosynthese über den gleichen operativen Zugang möglich als auch vor einer Marknagelosteosynthese über einen gesonderten $\mathrm{Zu}$ gang. Aufgrund der Immunsuppression der Patienten durch Chemo- und Strahlentherapie und die Grunderkrankung selbst sollte der Knochenzement eine Antibiotikazugabe enthalten.

Endoprothese. Die Implantation einer zementierten Endoprothese sollte bei gelenknahen Frakturen erfolgen, bei denen eine sichere Fixation der Osteosynthese zum Gelenk hin nicht mehr möglich ist. Eine Standardhüftprothese ist dann bei allen Läsionen des Hüftkopfs und Schenkelhalses indiziert (- Abb.4a-c, [3]). Bei Osteolysen unterhalb des Trochantermassivs oder weiteren Metastasen der Femurdiaphyse können Langschaftprothesen zum Einsatz kommen. Die Frage, ob eine Hemi- oder Totalarthroplastik verwendet werden sollte, wird kontrovers diskutiert. In Übereinstimmung mit Capanna u. Campanacci [3] verwenden wir in der Regel eine Hemiarthroplastik mit einem Duokopf, da hierbei die höchste Luxationssicherheit besteht. Nur bei Vorliegen von ausgeprägten arthrotischen Veränderungen oder einer simultanen Osteolyse des Acetabulums erfolgt die Implantation einer Hüftpfanne (bei einer Osteolyse des Acetabulums gegebenenfalls mit einer Pfannendachplastik) [3]. Capanna u. Campanacci [3] empfehlen bei diesen Patienten zur Vermeidung einer Luxation die Implantation einer Schnapppfanne oder eines Großkopfes (28-32 mm).

Wie großzügig die Indikation zum endoprothetischen Ersatz insbesondere bei Frakturen des proximalen Femurs gestellt wird, ist von Operateur zu Operateur höchst unterschiedlich. Größere Studien wie die von Wedin et al. [14] favorisieren insbesondere bei Frakturen des

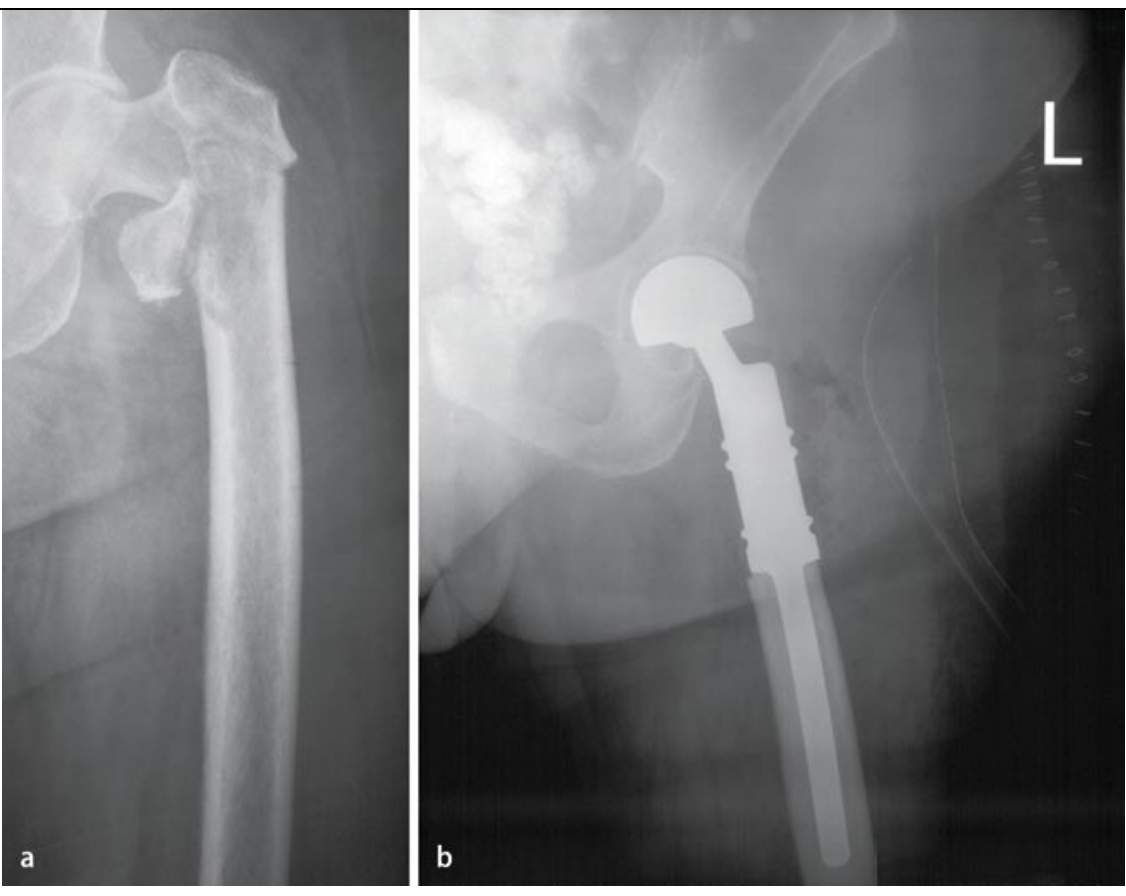

Abb. $5 \Delta$ a Anterio-posteriores Röntgenbild einer 75-jährigen Patientin mit einer pathologischen Fraktur bei singulärer Frühmetastase eines Mammakarzinoms. Die zugrunde liegende Osteolyse reicht bis subtrochantär, b postoperatives Röntgenbild, welches eine zementierte Tumorendoprothesenversorgung mitsamt einem Duokopf zeigt

proximalen Femurs die Implantation einer Endoprothese, da eine solche den betreffenden Skelettabschnitt komplett ersetzt, also nicht auf eine Frakturheilung angewiesen ist. In ihrem Patientenkollektiv versagten 22 von 162 (14\%) osteosynthetischen Stabilisierungen aufgrund von Pseudarthrosen, Tumorprogress und Infektionen im Mittel 8 Monate nach der Operation. Demgegenüber erfolgte bei nur einem von 52 Patienten mit einer Endoprothese eine Revisionsoperation. Yazawa et al. [15] ermittelten bei einer ausgedehnten peritrochantären Osteolyse ebenfalls bei $23 \%$ der Patienten das Versagen einer Osteosynthese im Bereich des proximalen Femurs. Sie kommen daher zu dem Schluss, dass diese Patienten bevorzugt mit einer Endoprothese versorgt werden sollten. In der Studie von Nathan et al. [13] erfolgte ebenfalls bei $62 \%$ der $155 \mathrm{~Pa}$ tienten mit einer Skelettmetastase die Implantation einer Endoprothese. Reoperationen waren zumeist aufgrund einer lokalen Tumorprogression notwendig, welche in der Mehrzahl der Fälle die Stabilität der Rekonstruktionsmethode tangierte. Von 96 Patienten mit einer endoprothetischen Rekonstruktion benötigten 5 (5,2\%) eine Revisionsoperation.

\section{Risikofaktoren}

In der Studie von Wedin et al. [14] zeigte sich tendenziell, dass in kleineren Krankenhäusern mit geringeren Fallzahlen häufiger osteosynthetische Versorgungen zum Einsatz kamen und diese in der Folge auch häufiger versagten. Die Autoren führen dies auf die Tatsache zurück, dass in diesen Einrichtungen Schwierigkeiten bei der Prognoseabschätzung und der Frakturheilung der Patienten vorliegen. Das Hauptproblem der Behandlung der pathologischen Fraktur liegt in der Abschätzung der Überlebenszeit der Patienten. In ihrer Studie überlebten 18\% der Patienten über 2 Jahre. Speziell in diesen Fällen sollte der möglichst weiten Tumorresektion und Tumorprothesenimplantation der Vorzug gegeben werden, da bei osteosynthetischer Versorgung und fehlender Frakturheilung häufig Materialversagen auftritt. Somit stellt für ein Implantatversagen nicht die Operationsmethode an sich den größten Risikofaktor dar, sondern - so paradox dies klingen mag - die Operationsmethode in Kombination mit dem Überleben der Patienten [11].

Tumorendoprothesen sind bei ausgedehnten Osteolysen erforderlich und sollten im Fall eines proximalen Femur- 
ersatzes in der Regel ebenfalls mit einem Duokopf versorgt werden ( $\mathbf{\bullet}$ Abb. 5a, b). In unserem eigenen Patientenkollektiv haben wir keine Luxation einer solchen Versorgung feststellen müssen. Im Fall einer Pfannenimplantation mussten unsere $\mathrm{Pa}$ tienten jedoch eine Bettruhephase einhalten, um einen Luxationsschutz zu gewährleisten. Andere Autoren versorgten die Patienten primär mit einer Orthese [3, 11]. Wir selbst verwenden seit über einem Jahr die Avantage ${ }^{\circledR}$-Pfanne (Biomet, Berlin), welche trotz der fest implantierten Pfannenkomponente aufgrund ihres tripolaren Aufbaus einen sehr guten Luxationsschutz erbringt.

Neben der Prothesenluxation stellt die periprothetische Infektion die häufigste und gravierendste Komplikation dar [9]. Eine Prothesenexplantation und Zementplatzhalterimplantation bedeutet hierbei für die Patienten eine starke Beeinträchtigung der Lebensqualität. Wir verwenden daher bei Patienten mit einem hohen Infektionsrisiko eine silberbeschichtete, antimikrobiell wirksame Tumorendoprothese $[10]$.

\section{Fazit für die Praxis}

Die Behandlung der pathologischen Fraktur unterscheidet sich grundlegend von der traumatisch bedingten, nichtpathologischen Fraktur. Bis zum Beweis des Gegenteils muss bei einer nur durch ein Minimaltrauma verursachten Fraktur eine pathologische Stabilitätsminderung des Knochens ausgeschlossen werden. Hierbei muss immer ein standardisierter diagnostischer Algorithmus eingehalten werden. Eine bioptische Sicherung ist zwingend erforderlich, sofern nicht bereits eine bekannte Skelettmetastasierung besteht. Bei der Diagnose eines primär malignen Knochentumors sollte die Überweisung an ein Referenzzentrum erfolgen. Bei Vorliegen einer Skelettmetastase hängt die Wahl des Therapieverfahrens unter anderem von der Lokalisation der Fraktur, der noch erhaltenen Knochensubstanz, der Strahlensensibilität des Tumors und entscheidend von der Prognose der Erkrankung ab. Die Abschätzung der Prognose ist jedoch für den Einzelfall höchst schwierig. Das größte Risiko für eine Revisionsope- ration stellt eine gute Prognose des $\mathrm{Pa}$ tienten dar; dies sollte in der Therapiewahl bedacht werden. Patienten mit einer guten Prognose und einer hüftgelenksnahen Fraktur sollten mit einer Endoprothese versorgt werden. Patienten mit einer nur kurzen Überlebenszeit sollten - falls eine ausreichende Knochensubstanz vorliegt - osteosynthetisch versorgt werden.

\section{Korrespondenzadresse}

\section{Dr. J. Hardes}

Klinik und Poliklinik für Allgemeine Orthopädie, Universitätsklinikum Münster Albert-Schweitzer-Str. 33, 48149 Münster hardes@uni-muenster.de

Interessenkonflikt. Der korrespondierende Autor gibt an, dass kein Interessenkonflikt besteht.

\section{Literaturverzeichnis}

1. Althausen $P$, Althausen $A$, Jennings LC, Mankin HJ (1997) Prognostic factors and surgical treatment of osseous metastases secondary to renal cell carcinoma. Cancer 80: 1103-1109

2. Bacci G, Longhi A, Versari M et al. (2006) Prognostic factors for osteosarcoma of the extremity treated with neoadjuvant chemotherapy: 15-year experience in 789 patients treated at a single institution. Cancer 106: 1154-1161

3. Capanna R, Campanacci DA (2001) The treatment of metastases in the appendicular skeleton. Journal Bone Joint Surg Br 83: 471-481

4. Coleman RE (2006) Clinical features of metastatic bone disease and risk of skeletal morbidity. Clin Cancer Res 12 (20): 6243s-6249s

5. Dürr HR, Maier M, Pfahler M et al. (1999) Surgical treatment of osseous metastases in patients with renal cell carcinoma. Clin Orthop 367: 283-290

6. Enneking WF (1986) A system of staging musculoskeletal neoplasms. Clin Orthop 204: 9-24

7. Gainor BJ, Buchert P (1983) Fracture healing in metastatic bone disease. Clin Orthop 178: 297

8. Hansen BH, Keller J, Laitinen M et al. (2004) The Scandinavian Sarcoma Group Skeletal Metastasis Register. Survival after surgery for bone metastases in the pelvis and extremities. Acta Orthop Scand Suppl 75: 11-15

9. Hardes J, Gebert C, Schwappach A et al. (2006) Characteristics and outcome of infections associated with tumor endoprostheses. Arch Orthop Trauma Surg 126: 289-296

10. Hardes J, Ahrens H, Gebert C et al. (2007) Lack of toxicological side-effects in silver-coated megaprostheses in humans. Biomaterials 28: 28692875

11. Jacofsky DJ, Haidukewych GJ (2004) Management of pathologic fractures of the proximal femur. State of the Art. J Orthop Trauma 18: 459-469

12. Mutschler W, Wirbel R (1997) Pathologische Frakturen. Unfallchirurg 100: 410-429

13. Nathan SS, Healy JH, Mellano D et al. (2005) Survival in patients operated on for pathologic fracture: implications for end-of-life orthopedic care. J Clin Oncol 23: 6072-6082
14. Wedin R, Bauer HCF, Rutqvist LE (2001) Surgical treatment for skeletal breast cancer metastases. A population-based study of 641 patients. Cancer 92: 257-262

15. Yazawa Y, Frassica FJ, Chao EYS et al. (1990) Metastatic bone disease. A study of the surgical treatment of 166 pathologic humeral and femoral fractures. Clin Orthop 251: 213-219 